\title{
Postoperative Acute Bilateral Irvine-Gass Syndrome following Uncomplicated Phacoemulsification Surgery with Trifocal IOL-Two Case Reports
}

\author{
Ishtiaque Anwar ${ }^{*}$, Sharah Rahman ${ }^{2}$ (D), Sofia Akhter ${ }^{3}$, Shah Noor Hassan ${ }^{4}$, Md. Sanwar Hossain ${ }^{5}$, \\ Siddiqur Rahman', Anisur Rahman7
}

${ }^{1}$ Glaucoma and Cataract Department, Bangladesh Eye Hospital and Institute, Dhaka, Bangladesh

${ }^{2}$ Cornea and Cataract Department, Bangladesh Eye Hospital and Institute, Dhaka, Bangladesh

${ }^{3}$ Clinical and Research Coordinator, Dhaka Eye Care Hospital, Dhaka, Bangladesh

${ }^{4}$ Vitreo-Retina Department, Bangladesh Eye Hospital and Institute, Dhaka, Bangladesh

${ }^{5}$ Ophthalmology Department, Dr. Sirajul Islam Medical College, Dhaka, Bangladesh

${ }^{6}$ Northern International Medical College, Dhaka, Bangladesh

${ }^{7}$ Dhaka Medical College and Hospital, Dhaka, Bangladesh

Email: *ishtiaqueanwar1976@gmail.com

How to cite this paper: Anwar, I., Rahman, S., Akhter, S., Hassan, S.N., Hossain, M.S., Rahman, S. and Rahman, A. (2022) Postoperative Acute Bilateral Irvine-Gass Syndrome following Uncomplicated Phacoemulsification Surgery with Trifocal IOL-Two Case Reports. Open Journal of Ophthalmology, $12,34-43$

https://doi.org/10.4236/ojoph.2022.121004

Received: November 1, 2021

Accepted: January 26, 2022

Published: January 29, 2022

Copyright $\odot 2022$ by author(s) and Scientific Research Publishing Inc. This work is licensed under the Creative Commons Attribution International License (CC BY 4.0).

http://creativecommons.org/licenses/by/4.0/

\begin{abstract}
The case studies are about atypical bilateral, postoperative Cystoid Macular Edema (CME) in two patients who underwent bilateral uncomplicated phacoemulsification surgery with a trifocal Intraocular Lens (IOL). In postoperative follow-up, both the cases presented with complaints of bilateral blurring of vision. Irvine-Gass syndrome has been diagnosed after evaluation of the retina by fundoscopy and OCT macula. Posterior subtenon steroid injection has been prescribed and given. Regular OCT examinations indicated substantial edema improvement. Subsequent improvement in visual acuity was also noted in both cases. Cystoid macular edema can happen in any case irrespective of the quality of IOL. The visual outcome is satisfactorily responsive towards steroid medication.
\end{abstract}

\section{Keywords}

Irvine-Gass Syndrome, Cystoid Macular Edema (CME), NSAID, Postoperative CME

\section{Introduction}

A cataract is a common ocular phenomenon worldwide for older people. Pha- 
coemulsification is the most modernized technique of cataract surgery and is commonly performed in ophthalmic practice. Though it has reduced the chance of surgical injury to the eye, unlike any other surgical procedures, it may produce complications. The pseudophakic cystoid macular edema is a severe and rare complication of uncomplicated cataract surgery that can cause transient or even permanent vision loss [1]. The cause of cystoid macular edema is multifactorial. Its development has various causative factors, such as the history of uveitis, diabetes mellitus, complicated cataract surgery, inflammation, vascular instability or vitreomacular traction, and light toxicity [2]. Cystoid Macular Edema (CME) develops due to extracellular fluid accumulation in the retina's outer plexiform and inner nuclear layers. This fluid accumulation is caused by the inflammatory process, which causes the breakdown of the physiological blood-retinal barrier. Surgical manipulations of the anterior chamber result in the release of arachidonic acid from uveal tissue. Thus, the inflammatory response is triggered by the production of leukotrienes and prostaglandin via the lipo-oxygenase and the cyclo-oxygenase pathway, respectively [1]. The pathogenesis of the Cystoid Macular Edema is not fully understood. Still, its diagnosis can be quickly confirmed by clinical and angiographic examination like fundoscopy, Fundus Fluorescein Angiography (FFA), and Optical Coherence Tomography (OCT) of the macula. According to reports, it is a relatively unusual disease, with just $0.2 \%$ - $2 \%$ of post-surgical patients developing this problem [3] [4]. The development time of cystoid macular edema may vary within 6 - 12 postoperative weeks of cataract surgery.

The purpose of this article is to notify the possibility of Irvine-Gass syndrome following phacoemulsification surgery, even if a premium trifocal lens is used and the procedure was uneventful.

\section{Case Report}

\subsection{Case 1}

A 51 years old male, the non-diabetic, non-hypertensive patient, underwent uncomplicated and uneventful phacoemulsification surgery in both eyes, one day apart due to bilateral age-related cataract in November 2020. Trifocal IOL (Panoptics IOL by Alcon) was implanted in both eyes. Preoperative examination revealed BCVA in both eyes (BE) was 6/18 in Snellen's chart and 0.48 in the LogMAR chart. IOL Master measured the axial length, anterior chamber depth and lens thickness of the right eye about $23.34 \mathrm{~mm}, 3.40 \mathrm{~mm}, 4.28 \mathrm{~mm}$ and the left eye approximately $23.26 \mathrm{~mm}, 3.31 \mathrm{~mm}, 4.38 \mathrm{~mm}$, respectively. Swept-source OCT checked preoperative retinal morphology. Macular thickness was $246 \mu \mathrm{m}$ in the right eye and $245 \mu \mathrm{m}$ in the left eye. He had no significant ocular history of uveitis or retinal disease but gave the history of having hyperthyroidism as medical history. The patient underwent uneventful phacoemulsification surgery with posterior chamber Trifocal Intraocular lens (Panoptics) implantation within the bag under topical anaesthesia in both eyes. The IOL power was 22.0 D in RE and 22.50 in L.E.. The surgery was done by an experienced surgeon. The immediate postoperative evaluation was favourable. BCVA in the first Post-Operative Day (POD) 
was 6/6 partial in Snellen's chart and 0.00 in the LogMAR chart in both eyes. The anterior segment and the anterior chamber were clear; there was no sign of inflammation or abnormality seen in the vitreous. The patient was discharged from the hospital. He came for postoperative follow-up after one month with complaints of gradual blurring of vision in both eyes.

In both eyes, the BCVA drop down to 6/18 in Snellen's chart and 0.48 in the LogMAR chart. Central Corneal Thickness (CCT) was $560 \mu \mathrm{m}$ in the right eye and $570 \mu \mathrm{m}$ in the left eye. Intra-Ocular Pressure (IOP) was $13 \mathrm{~mm} \mathrm{Hg}$ and 11 $\mathrm{mm} \mathrm{Hg}$ in the right and left eye, respectively. Investigations had done to find out the aetiology behind this post-surgical reduced vision. RFNL thickness and OCT macula had done for both eyes. RFNL thickness reports didn't reveal any abnormality, but a remarkable elevation in macular thickness is shown in Figure 1. The report showed the central macular thickness was $518 \mu \mathrm{m}$ in the right eye and 475 $\mu \mathrm{m}$ in the left eye.

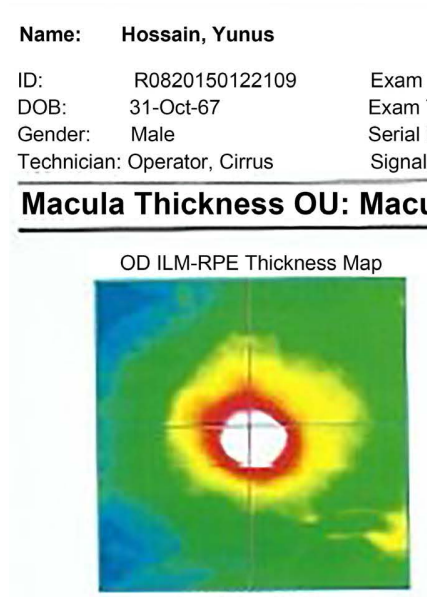

Fovea: 262,61
OD OS Time: $\quad$ 5:17 PM 5:18 PM erial Number: 6000-6735 6000-6736 Signal Strength: $5 / 10$ $6 / 10$
यहार

CZM

128

OD O O OS
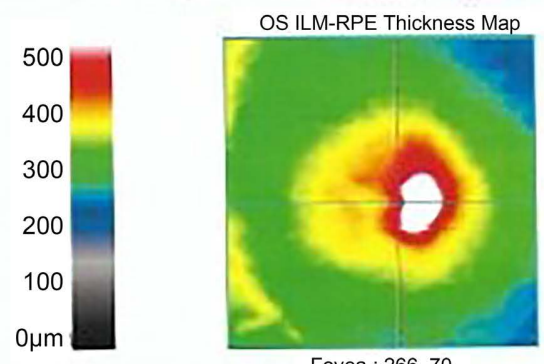

Fovea : 266,70

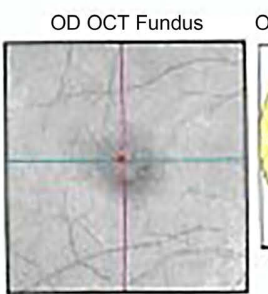

OD ILM-RPE Thickness
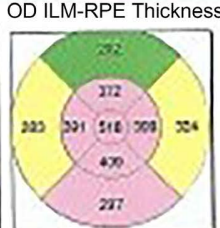

$2 \pi 7$
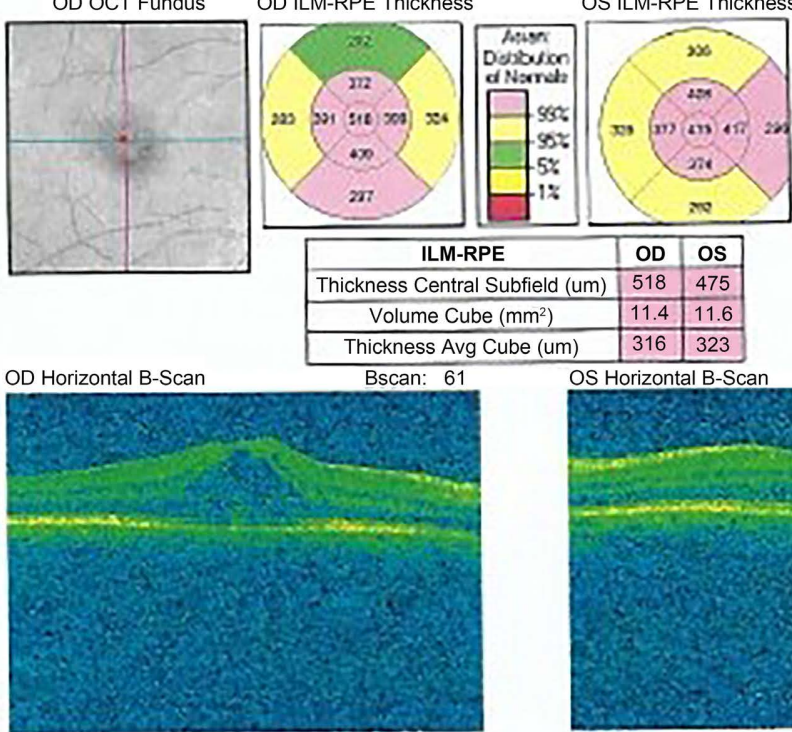

OS ILM-RPE Thickness
OS OCT Fundus
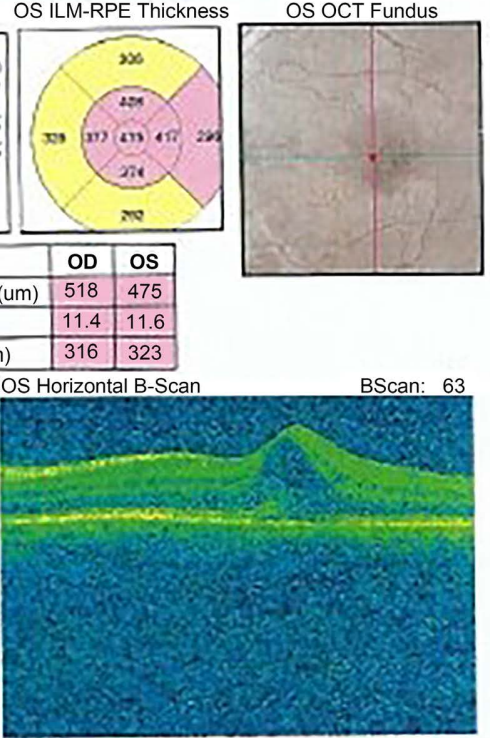

Figure 1. OCT Macula showed the central macular thickness was $518 \mu \mathrm{m}$ in the right eye and $475 \mu \mathrm{m}$ in the left eye on 10 January 2021. 
The case was diagnosed as bilateral cystoid macular edema (CME)/Irvine-Gass syndrome. The patient was prescribed NSAID, artificial tear, and antibiotic eye drop and was referred to the retina specialist for posterior subtenon administration of injectable steroid. The patient was counselled adequately before the initiation of steroid injection and about its possible side effects. Injection Triamcinolone Acetonide has been given in both eyes on two consecutive days. Triamcinolone acetonide $1 \mathrm{ml}, 40 \mathrm{mg} / \mathrm{ml}$, administered at the posterior sub tenon space with a $26 \mathrm{G}$ needle bevel down following the curve of the globe superotemporally. We did not notice any side effects of the subtenon injections. Repeat OCT macula was done to evaluate the effect of steroids on macula after one week. The repeat OCT showed a significant reduction in macular thickness, $292 \mu \mathrm{m}$ in the right eye and $307 \mu \mathrm{m}$ in the left eye (Figure 2). The patient's vision was restored

\begin{tabular}{|c|c|c|c|c|c|c|}
\hline Name: & Hossain, Yunus & & $O D$ & os & & \\
\hline ID: & R0820150122109 & Exam Date: & 18-Jan-21 & 18-Jan-21 & CZM & \\
\hline DOB: & $31-$ Oct-67 & Exam Time: & 4:53 P M & 4.64 PM & & \\
\hline Gender: & Male & Serial Number: & $6000-6735$ & $6000-6736$ & & \\
\hline Technician: & n: Operator, Cirrus & Signal Strength & h: $7 / 10$ & $6 / 10$ & & \\
\hline
\end{tabular}

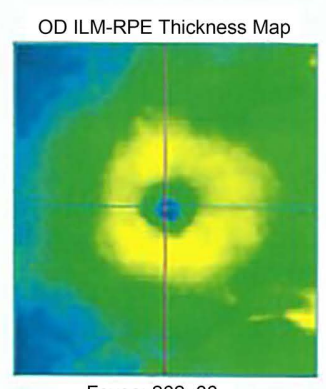

Fovea: 262,66

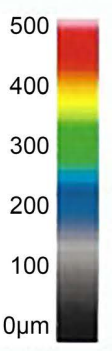

$0 \mu \mathrm{m}$

OS ILM-RPE Thickness Map

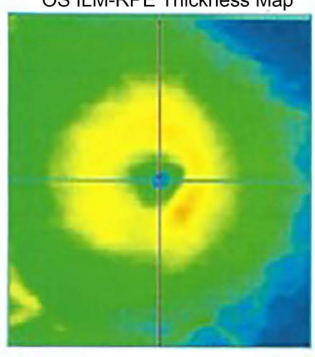

Fovea : 254,65

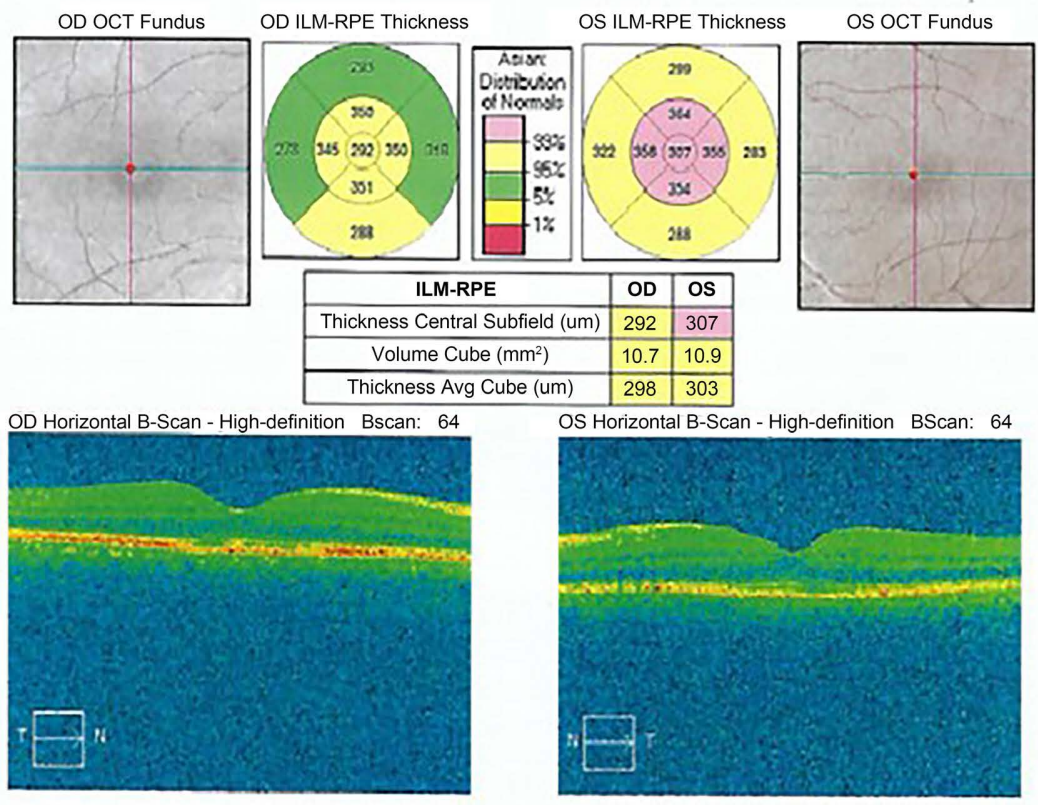

Figure 2. OCT Macula showed the central macular thickness was $292 \mu \mathrm{m}$ in the right eye and $307 \mu \mathrm{m}$ in the left eye on 18 January 2021 after the posterior sub tenon injection. 
with BCVA 6/6p, 0.00 in the right eye, and 6/6, 0.00 in the left eye by Snellen's and LogMAR charts. The injectable steroid has been proven to have a good response after one week. The patient was maintaining BCVA of 6/6, 0.00 by Snellen's and LogMAR charts in both eyes on the subsequent follow up of 1 month and six months.

\subsection{Case 2}

A 56 years old male patient underwent uneventful phacoemulsification surgery within bag Trifocal IOL (Panoptics) implantation. He came for follow-up on 30 December 2020 with the chief complaints of the dimness of vision in both eyes for one week. His I.O.P. was $20 \mathrm{~mm} \mathrm{Hg}$ in the right eye and $17 \mathrm{~mm} \mathrm{Hg}$ in the left eye. Unaided visual acuity was 6/18 (Snellen chart), 0.48 (in Log M.A.R. chart) in both eyes. The patient was hypertensive and non-diabetic. On funduscopy, the macula was dull in both eyes, which were suggestive of suspected macular edema. OCT macula was done on that day to confirm the diagnosis. On OCT, the central macular thickness was $534 \mu \mathrm{m}$ in the right eye and 542 in the left eye shown in Figure 3 and Figure 4.

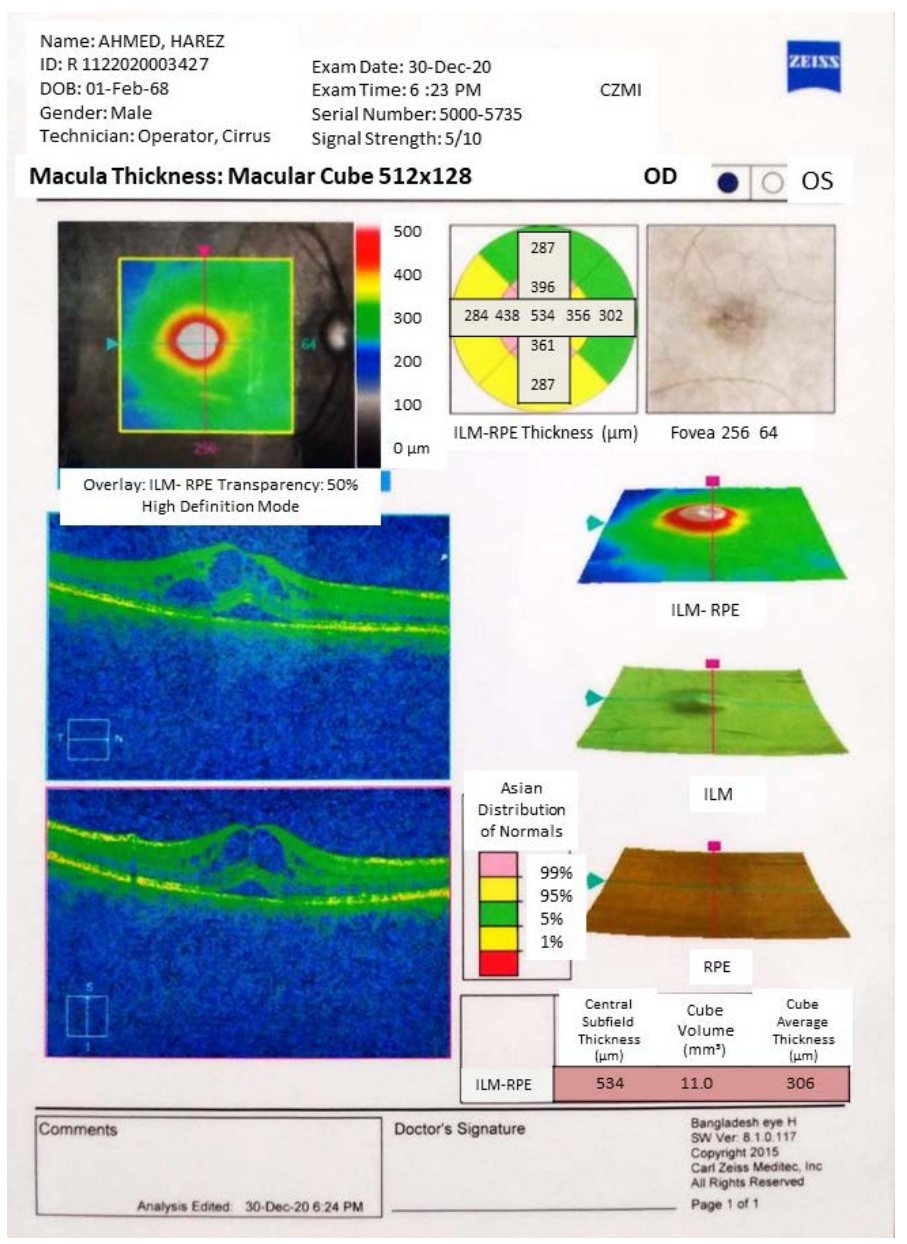

Figure 3. OCT macula on 30 December 2020 showed that the central macular thickness was $534 \mu \mathrm{m}$ in the right eye. 


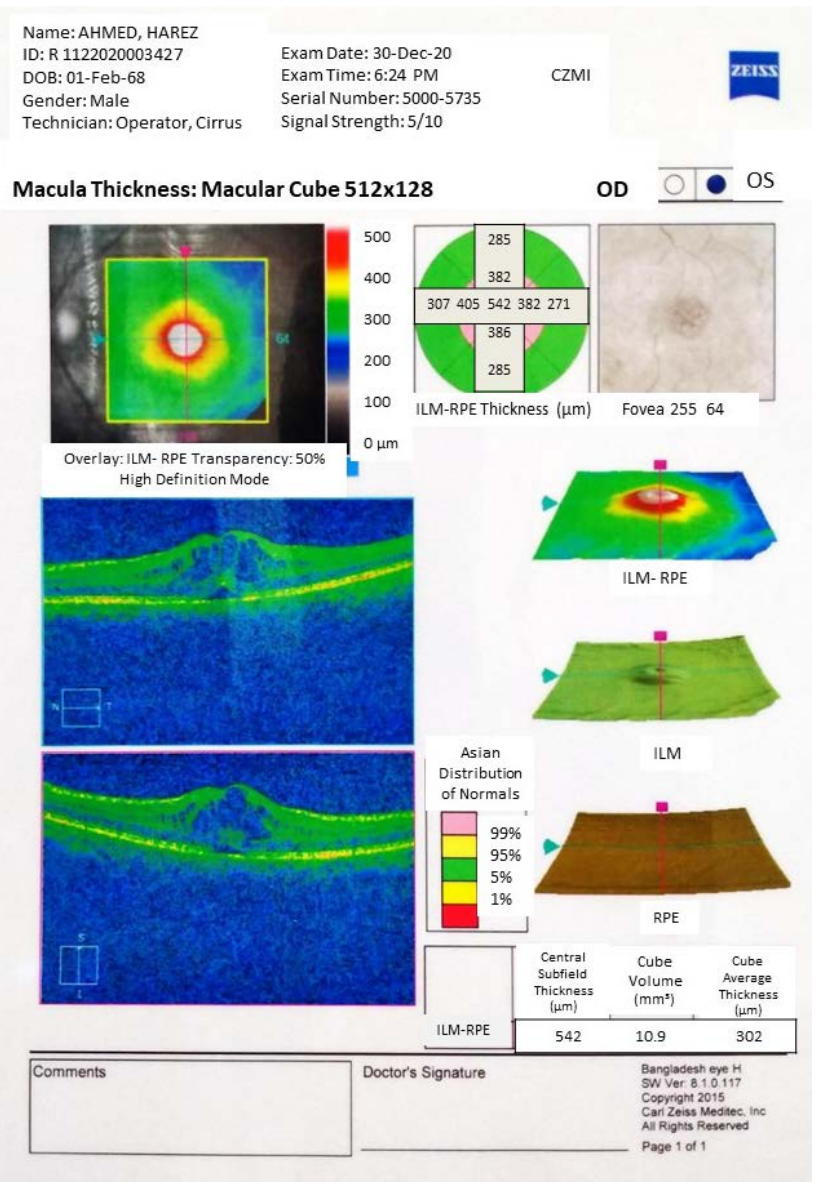

Figure 4. OCT macula on 30 December 2020 showed that the central macular thickness was $542 \mu \mathrm{m}$ in the left eye.

We diagnosed Irvine Gass Syndrom or Pseudophakic Cystoid Macular Edema based on the significant intraretinal and subretinal fluid deposition detected on the macular imaging. The patient was advised with a posterior subtenon steroid injection. The patient and the attendant were counselled adequately about the treatment procedure's process, prognosis, and possible side effects. Posterior subtenon injection Triamcinolone Acetonide has been given in both eyes in two consecutive days. The patient was advised to return in three weeks for a follow-up OCT to examine the effect of the steroid on the macula. On follow-up, after three weeks (23 January 2021), the patient's visual acuity improved to 6/6 in the Snellen chart and 0.00 in the LogMAR chart in both eyes. IOP were 12 and $16 \mathrm{~mm} \mathrm{Hg}$ in the right and left eyes, respectively. On fundus examination, the discs were normal, and there was no sign of macular edema in both right and left eyes. To date, the patient is maintaining visual acuity of $6 / 6$.

Repeat OCT macula on that day (23 January 2021) showed reduced central macular thickness. The macular thickness in OCT was $237 \mu \mathrm{m}$ in the right eye and $236 \mu \mathrm{m}$ in the left eye, shown in Figure 5 and Figure 6. Substantial visual improvement was observed by administering steroids in the posterior sub tenon space. 


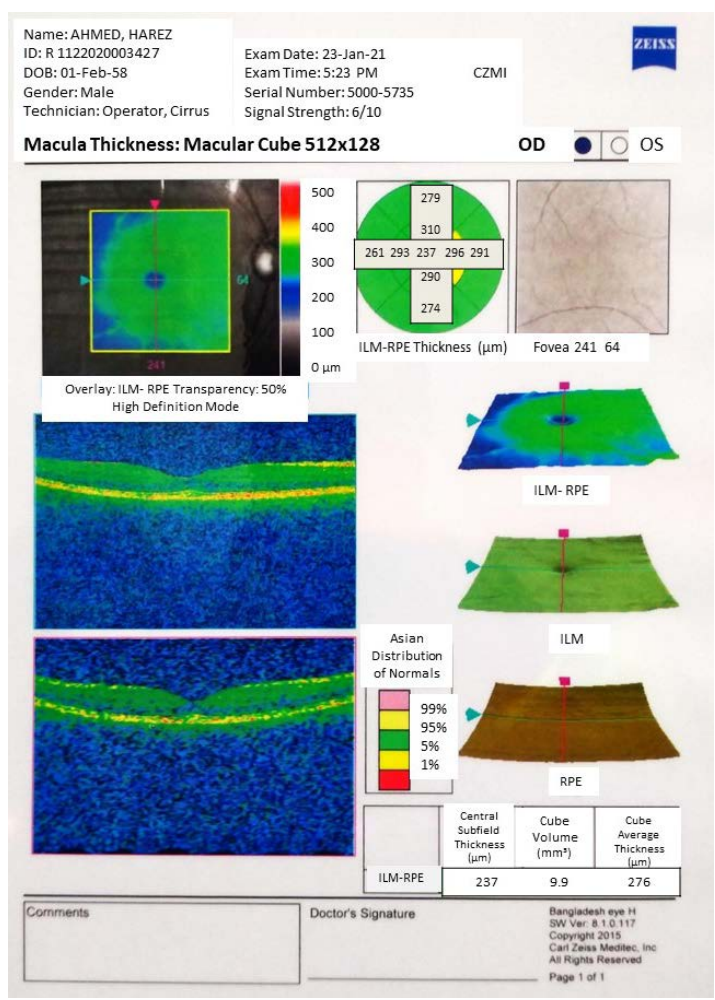

Figure 5. Repeat OCT Macula on 23 January 2021 showed the central macular thickness was $237 \mu \mathrm{m}$ in the right eye after the posterior sub tenon injection.

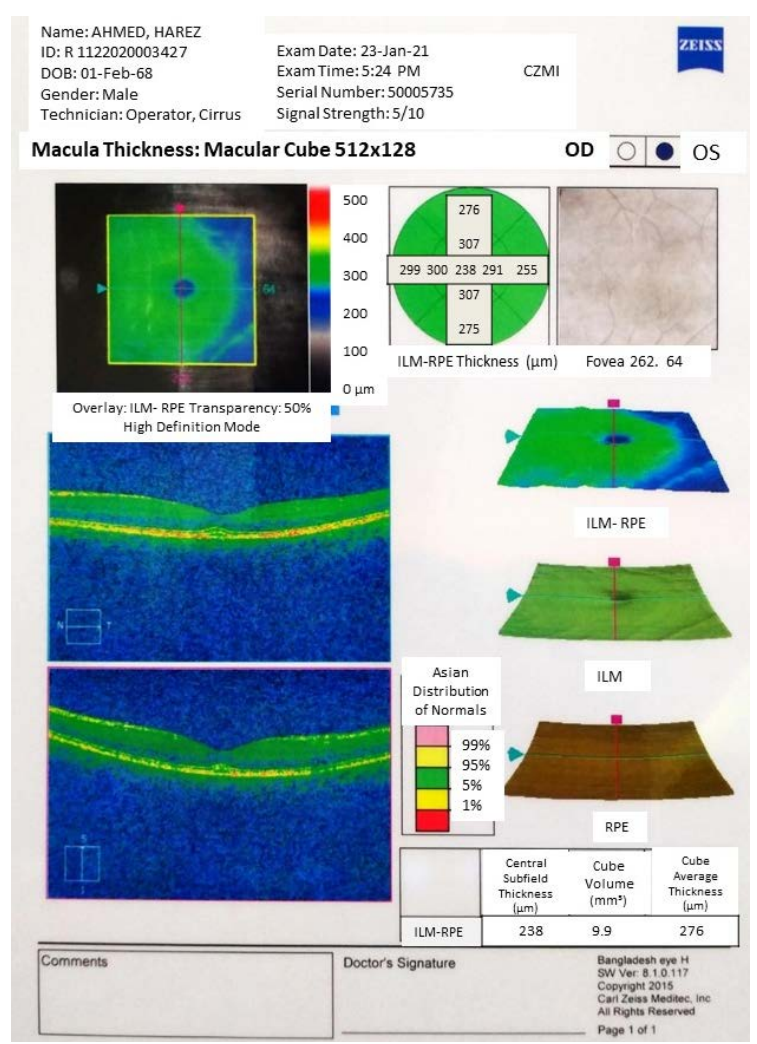

Figure 6. Repeat OCT Macula on 23 January 2021 showed the central macular thickness was $236 \mu \mathrm{m}$ in the left eye after the posterior sub tenon injection. 
In both cases, Irvine-Gass syndrome occurred, irrespective of the use of a premium IOL (Trifocal Panoptics IOL), which was managed by administering posterior sub tenon injections.

\section{Discussion}

CME is a primary cause of reduced vision after cataract surgery. Many risk factors like prolonged surgery, use of topical prostaglandin analogue, diabetes etc., can cause Irvine-Gas syndrome. The pathophysiological mechanism responsible for post-operative CME is also not explained yet. Irvine proposed that early changes occur due to direct macular traction from the prolapsed vitreous [5]. Scarpa also revealed that macular traction on the iris or the ciliary body might initiate the inflammatory mechanism that ultimately causes CME [6]. Although the exact aetiology of CME is not known, it seems, inflammation has a strong influence on its development. Surgical manipulation also upgrades the inflammatory mediator, leading to the blood-retinal barrier breakdown and increasing permeability. This inflammation is associated with abnormal intracellular and extracellular fluid accumulation, resulting in classic cystic changes in the macula's outer plexiform, inner nuclear layer, and retinal thickening [7].

CME can cause unexpected compromised postoperative loss of vision even after uncomplicated cataract surgery. The differential diagnosis can take into consideration to confirm the cause of diminished vision after phacoemulsification. TASS (Toxic Anterior Segment Syndrome) is a rare, acute, and severe intraocular inflammation that usually takes place within 12 - $48 \mathrm{hrs}$. Our patient had no increased intraocular pressure, diffuse corneal edema, hypopyon, or photophobia. So TASS can be excluded. Hypotonus maculopathy is another complication that may cause postoperative dimness of vision. This possibility can also be excluded as the patient's intraocular pressure was within the normal limit. Retinal vein occlusion and vitreomacular traction could be potential causes of postoperative visual loss [6]. Diabetes also plays a role in the pathogenesis of CME [8].

In mild cases, postoperative CME resolves spontaneously and requires no treatment. Some eyes resist common medical treatment strategies, including systemic or topical non-steroidal anti-inflammatory drugs [9]. Intravitreal application of triamcinolone acetonide injection has been used to treat postoperative CME for a long [10].

In our case studies, both patients developed CME after uneventful phacoemulsification surgery. Postoperative OCT showed a significant change in macular thickness. Posterior Subtenon Injection (PST) has been given with a $26 \mathrm{G}$ needle bevel down along the curvature of the globe supero temporally. PST injection showed remarkable vision improvement. The results have been documented by post-procedure OCT macula and manifest visual refraction, which showed significant improvement with decreased edema and increased visual acuity. 


\section{Conclusion}

Irvine-Gass syndrome can happen following uncomplicated phacoemulsification surgery, irrespective of using a premium Intraocular Lens. To date, to the best of our knowledge, back to back cases of Irvine-Gass syndrome after uneventful phacoemulsification surgery with Trifocal Panoptics IOL were not reported. As a result, we have decided to report these two unusual incidents.

\section{Acknowledgements}

We are thankful to the patients' family for their kind consideration regarding the photographs for publication.

\section{Conflicts of Interest}

The authors declare no conflict of interest regarding the publication of this paper.

\section{References}

[1] Haleem, A., Rehman, A., Saleem, A., Memon, S., Memon, N., Fahim, M.F. (2017) Cystoid Macular Oedema after Phacoemulsification with and without Type 2 Diabetes Mellitus; A Hospital-Based Clinical Prospective Trial in Karachi. The Journal of the Pakistan Medical Association, 67, 395-399.

[2] Gulkilik, G., Kocabora, S., Taskapili, M. and Engin, G. (2006) Cystoid Macular Edema after Phacoemulsification: Risk Factors and Effect on Visual Acuity. Canadian Journal of Ophthalmology, 41, 699-703. https://doi.org/10.3129/i06-062

[3] Mentes, J., Erakgun, T., Afrashi, F. and Kerci, G. (2003) Incidence of Cystoid Macular Edema after Uncomplicated Phacoemulsification. Ophthalmologica, 217, 408-412. https://doi.org/10.1159/000073070

[4] Chaudhary, C., Bahadhur, H. and Gupta, N. (2015) Study of Cystoid Macular Edema by Optical Coherent Tomography following Uneventful Cataract Surgery. International Ophthalmology, 35, 685-691. https://doi.org/10.1007/s10792-014-9998-5

[5] Sheludchenko, V.M., Ronzina, I.A. and Galoyan, N.S. (2015) A Case of Bilateral Cystoid Macular Edema (Irvine-Gass) after Bilateral Multifocal Intraocular Lens Implantation. Vestnik Oftalmologii, 131, 82-89. https://doi.org/10.17116/oftalma2015131182-89

[6] Scarpa, G. (2011) Bilateral Cystoid Macular Edema after Cataract Surgery Resolved by Vitrectomy. European Journal of Ophthalmology, 21, 677-679. https://doi.org/10.5301/EJO.2011.6506

[7] Han, J.V., Patel, D.V., Squirrell, D. and McGhee, C.N.J. (2019) Cystoid Macular Oedema following Cataract Surgery: A Review. Clinical \& Experimental Ophthalmology, 47, 346-356. https://doi.org/10.1111/ceo.13513

[8] Panozzo, G., et al. (2020) Prevalence of Diabetes and Diabetic Macular Edema in Patients Undergoing Senile Cataract Surgery in Italy: The DIabetes and CATaract Study. European Journal of Ophthalmology, 30, 315-320. https://doi.org/10.1177/1120672119830578

[9] Wielders, L.H.P., Schouten, J. and Nuijts, R. (2018) Prevention of Macular Edema after Cataract Surgery. Current Opinion in Ophthalmology, 29, 48-53. https://doi.org/10.1097/ICU.0000000000000436 
[10] Boscia, F., Giancipoli, E., D’Amico, R.G. and Pinna, A. (2017) Management of Macular Oedema in Diabetic Patients Undergoing Cataract Surgery. Current Opinion in Ophthalmology, 28, 23-28. https://doi.org/10.1097/ICU.0000000000000328 